\title{
Multilayer emulsions stabilized by vegetable proteins and polysaccharides
}

\author{
César Burgos-Díaz ${ }^{1 *}$, Traudy Wandersleben ${ }^{2}$, Ana M. Marqués ${ }^{3}$, Mónica Rubilar ${ }^{1,4}$ \\ ${ }^{1}$ Agriaquaculture Nutritional Genomic Center, CGNA, Technology and Processes Unit, Temuco, Chile. \\ ${ }^{2}$ Agriaquaculture Nutritional Genomic Center, CGNA, Genomic and Bioinformatic Unit, Temuco, Chile \\ ${ }^{3}$ Laboratory of Microbiology, Faculty of Pharmacy, University of Barcelona. Barcelona, Spain. \\ ${ }^{4}$ Departament of Chemical Engineering, Scientific and Technological Bioresource Nucleus, BIOREN, Universidad de La \\ Frontera, Temuco, Chile
}

"Corresponding author: Tel.: +56 45 2744232; Fax: +56 452732402.

E-mail address: cesar.burgos@cgna.cl (C. Burgos-Díaz)

\begin{abstract}
There is great interest in the food, cosmetic and pharmaceutical industry in the use of proteins and polysaccharides as natural hydrocolloids to create novel emulsion systems with improved stability and functionality. For example, the electrostatic interaction between proteins and polysaccharides may be used to form oil-in-water (O/W) emulsions with multilayered interfacial membranes around oil droplets or multilayer emulsions. This type of emulsions have been developed using the layer-by-layer $(L b L)$ technique, which consists of direct adsorption of an oppositely charged polyelectrolyte layer (e.g. polysaccharides) on a primary layer of ionic emulsifiers (e.g. proteins). The polymeric structure and electrical charge of proteins make them a special class of compounds very suitable for its utilization in the $L b L$ technique. In recent years, the utilization of proteins as emulsifiers in food and pharmaceutical industry has been turning towards plants as a preferred alternative to animal-based sources. This article reviews the current understanding of the utilization of different vegetable proteins as emulsifier in order to stabilize $\mathrm{O} / \mathrm{W}$ multilayer emulsions systems. Additionally, it highlights some potential applications of the multilayer emulsion
\end{abstract}


technology in the industry, for improving the stability of emulsions to environmental stresses and for developing controlled or triggered release systems.

Key words: multilayer emulsions, vegetable proteins, polysaccharides, oil-in-water, layerby-layer

\section{Introduction}

Current technological development in industry demands new emulsifiers or methodologies to obtain stable emulsions, thus creating novel products or improving its shelf-live associated with an adequate delivery activity, efficiency and yield of active principles. Oilin-water emulsions $(\mathrm{O} / \mathrm{W})$ are widely used in the cleaning, cosmetics, pharmaceutical and food industries for encapsulating different bioactive compounds and increasing their solubility and stability. Homogenization of oily and aqueous phases is achieved in the presence of one or more emulsifiers. The emulsifier is adsorbed to the surface of just formed droplets reducing the interfacial tension and facilitating droplet disruption.

In recent years, proteins from animal and vegetables sources, have been considered as natural emulsifiers in different industrial processes [1]. Their biodegradability, compatibility and excellent characteristics are remarkable properties of these natural polymers. Moreover, industries in their search for protein ingredients have been turning towards plants as a preferred alternative to animal-based sources, e.g. in vegetarian diets, due to increased consumer concerns over the safety of animal-derived products [2*].

Many types of proteins can be used as emulsifiers due to their amphiphilic character, polymeric structure, and electrical charge characteristics [3]. The amphiphilic character means that they can be adsorbed to the droplet surfaces during homogenization. Despite 
their good features, protein-stabilized emulsions are highly sensitive to environmental stresses such as $\mathrm{pH}$, ionic strength and temperature, affecting encapsulated compounds [4**]. In this regards, it has been shown that these emulsions are particularly sensitive to $\mathrm{pH}$ and ionic strength. They tend to flocculate at $\mathrm{pH}$ values close to the isoelectric point of the adsorbed proteins and when the ionic strength exceeds a particular level, because the electrostatic repulsion between the droplets is then no longer sufficiently strong to overcome the various attractive interactions. This instability may limit their application in some commercial products [5]. As a way to overcome this disadvantage, the strategy is the incorporation of additional polysaccharide coating layers that stabilize the $\mathrm{O} / \mathrm{W}$ emulsions by means of electrostatic interaction with the protein layer. Along these lines, Guzey and McClements $\left[4^{* *}\right]$ convey that, one strategy to improve the physical stability of $\mathrm{O} / \mathrm{W}$ emulsions to environmental stresses is to form multilayer emulsions. Multilayer emulsions consist of various interfacial layers of proteins (emulsifier) and/or polysaccharides around oil droplets which are deposited using the $L b L$ electrostatic technique. $L b L$ has been shown to have potential applications in foods, including controlled/triggered release, stabilization of emulsions, processing and storage conditions. The production of stable multilayered emulsions using this technique depends on biopolymer properties (e.g., charge density, molecular weight and conformation), emulsifier layer thickness and bulk physicochemical conditions [1]. Additionally, multilayer emulsions have the potential to decrease lipid oxidation rates due to their ability to alter both the emulsion droplet charge and thickness of the interfacial region [6]. The electrical properties of the first layer of a multilayer emulsion are determined by the emulsifier, and they can therefore be controlled by selecting different types of emulsifiers [4**]. 
Several studies have shown that vegetable proteins can be used to prepare multilayer emulsions using the $L b L$ technique $[1,3,7]$. The use of vegetable proteins as emulsifier reflects the present "green" trend in the pharmaceutical, cosmetics and food industries. In food applications, vegetable proteins are known to be less allergenic compared to animal derived proteins $[2 *]$. This review presents the recent works dealing with the use of vegetable proteins in the formation of multilayer emulsions systems. The influence of the combination of different biopolymers, proteins, and polysaccharides on stability of $\mathrm{O} / \mathrm{W}$ multilayer emulsions, and the industrial applications in several industrial processes will be particularly discussed. Besides, the review is focused on the utilization of $L b L$ technique in the formation on multilayer emulsions. The information collected to prepare this revision follows the description of main published experimental studies in the last ten years.

\section{Vegetable proteins as emulsifier}

Proteins are commonly used in the food and pharmaceutical industry as emulsifiers in the stabilization of $\mathrm{O} / \mathrm{W}$ emulsions. These natural polymers present several advantages: biocompatibility, biodegradability, good amphiphilic and functional properties such as water solubility, emulsifying and foaming capacity. Proteins are amphiphilic compounds, and therefore, they are able to adsorb strongly at the oil-water interface, favoring emulsion formation [8]. The amount adsorbed and the conformation adopted at the oil-water interface will depend very much on the protein amino acid composition since adsorption occurs through hydrophobic groups present within their structure [9].

Nowadays, the utilization of vegetable proteins as emulsifiers has increased even surpassing the use of proteins from animal sources. In food applications, for example, vegetable proteins are known to be less allergenic compared to animal derived proteins. 
Vegetable proteins consist of several fractions: the major fraction is glutenin, followed by globulin fraction, albumin and prolamin [2*].

Among vegetable proteins used as emulsifiers, we can mainly find soy protein isolate, pea protein isolate, lupin protein isolate, broad beans and cereal proteins (such as wheat proteins) $[10,11]$. These types of proteins have been used to facilitate the formation, improve the stability, and provide specific physicochemical properties to emulsions [2*]. Many vegetable proteins are surface-active molecules that can be used as emulsifiers because of their ability to facilitate the formation, improve the stability and produce desirable physicochemical properties in $\mathrm{O} / \mathrm{W}$ emulsions [12*].

Despite their functional properties and benefits, emulsions stabilized by proteins are highly sensitive to environmental stresses such as $\mathrm{pH}$, ionic strength and temperature [13]. For example, at $\mathrm{pH}$ values close to the isoelectric point of protein and/or high salt concentration in the emulsion, the electrostatic repulsion of the protein adsorption layers decreases and therefore, coalescence and flocculation happen [13]. Besides, when emulsion is subjected to heat treatment, for pasteurization or sterilization purposes, flocculation happens because of the protein denaturation which holds the droplets together [14]. For this reason, several strategies have been developed to improve the stability of protein-stabilized emulsions to droplet flocculation induced by $\mathrm{pH}$ or ionic strength effects. For example, (1) the incorporation of multivalent counterions, such as $\mathrm{Ca}^{2+}, \mathrm{Fe}^{2+}$ or $\mathrm{Fe}^{3+}$, to emulsions systems; (2) the addition of ionic surfactants to protein-stabilized emulsion to change the $\mathrm{pH}$ dependence of the $\zeta$-potential of the droplets, thereby changing the range of $\mathrm{pH}$ values so that the emulsion is stable to flocculation [12*]; and, (3) the addition of electrically charged biopolymers to the surface of oppositely charged droplets to a protein-stabilized emulsion in order to increase its physical stability to environmental stresses [15*]. The $L b L$ 
electrostatic technique is normally utilized for forming this type of systems. This last topic will be explained in detail in section 3 and 4 of the present review.

\section{Design and preparation of multilayer emulsions}

Multilayer emulsions consist of small oil droplets, dispersed in an aqueous medium, surrounded by a multilayered interfacial membrane generally composed of an emulsifier (surfactant or protein) and a charged biopolymer (polysaccharides) (Figure 1) [7]. Emulsions containing oil droplets stabilized by protein-polysaccharide membranes are formed using $L b L$ deposition process. In this method, a primary emulsion is prepared by homogenizing oil and water phases in the presence of a positively or negatively charged emulsifier. The resulting primary emulsion is then mixed with an oppositely charged polyelectrolyte solution to create a secondary emulsion. The secondary emulsion is then mixed into another solution containing polyelectrolytes that have an opposite charge to the previous one to create a tertiary emulsion (Figure 2) [4**].

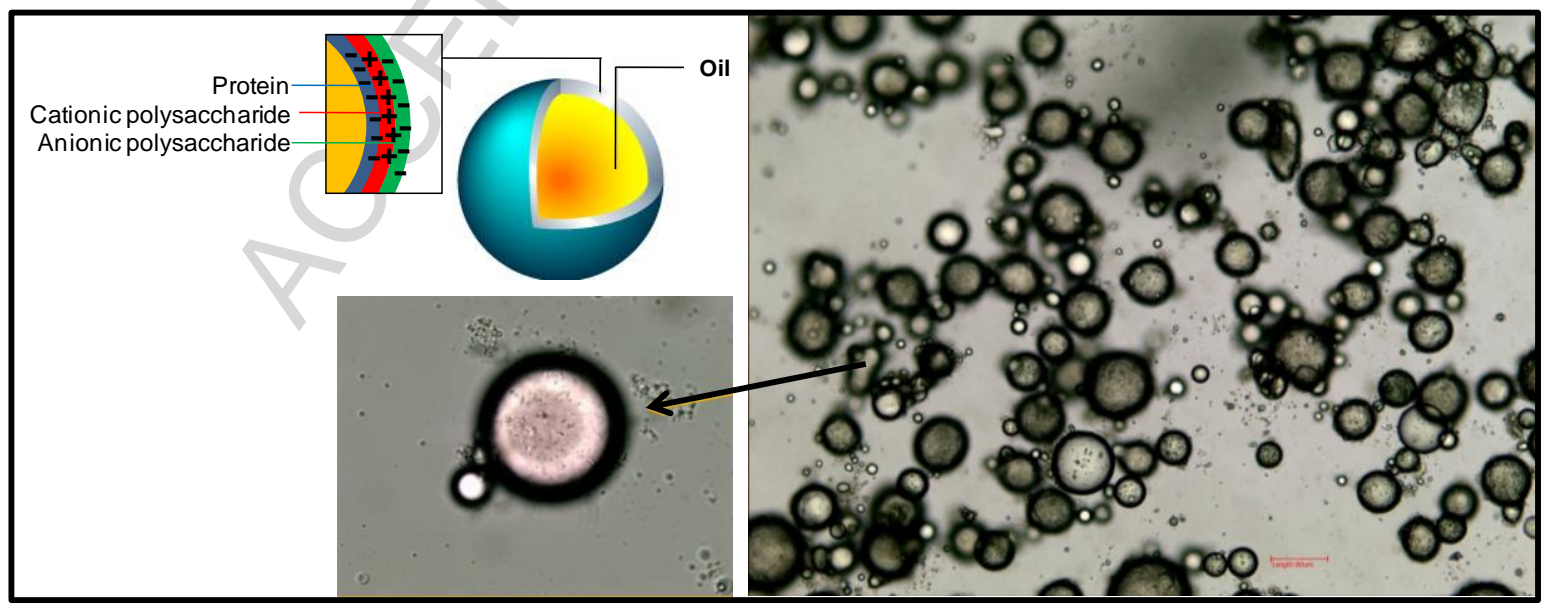

Figure 1: The figures correspond to optical micrograph pictures of secondary emulsion ( $5 \mathrm{wt} \%$ oil, $0.25 \mathrm{wt} \%$ lupin protein, and $0.06 \mathrm{wt} \%$ chitosan). 


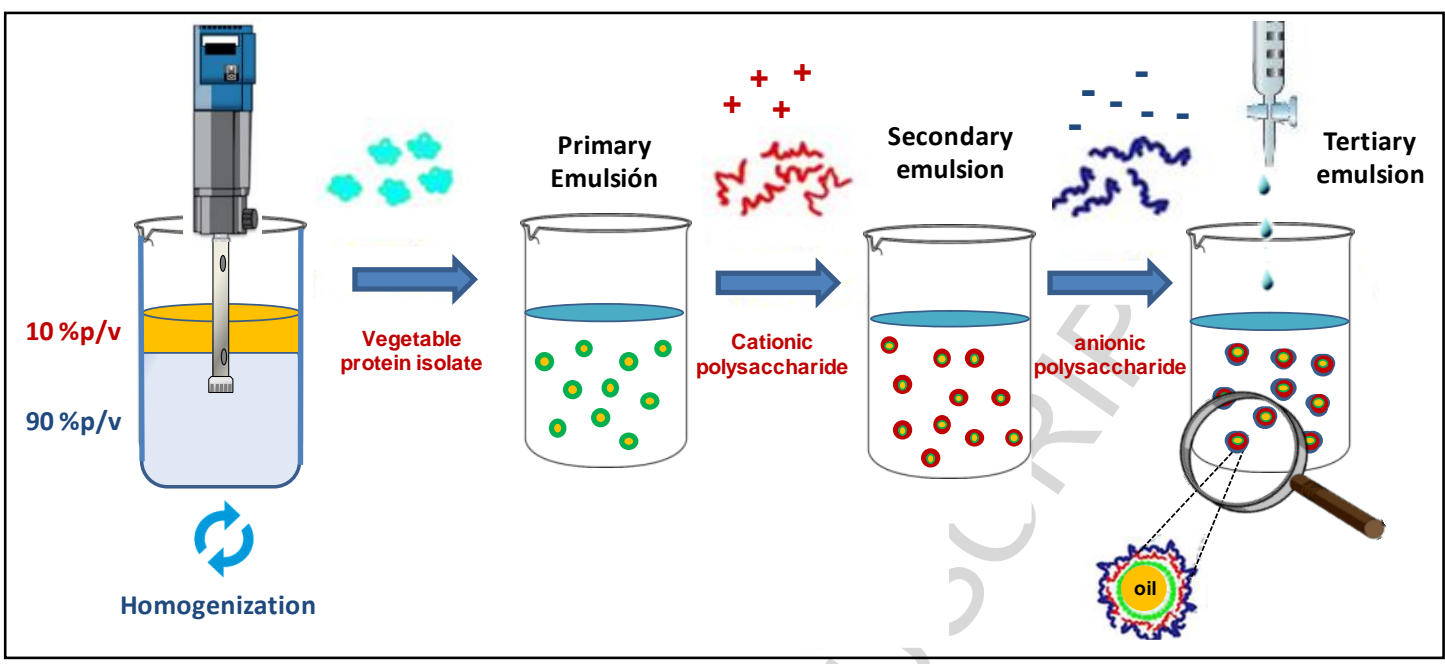

Figure 2: Schematic representation of the formation of a multilayer emulsion system. Figure adapted from Burgos-Díaz et al.[15*].

The $L b L$ deposition technique offers a promising way to prepare emulsions using electrostatic attraction of charged biopolymers to oppositely charged droplets [4**]. Emulsions prepared using this method have been shown to have enhanced stability in respect of ionic strength $\left[4^{* *}\right], \mathrm{pH}[16]$ and temperature [15*]. Moreover, this method provides a new powerful tool to improve resistance of food emulsions to extreme environmental stresses [4**].

According to Burgos-Díaz and coworkers [15*], to create stable multilayer emulsions with the required physicochemical properties, it is essential to choose a suitable combination of emulsifier and biopolymers. Thus, the ionic behavior (electrical charge) should be evaluated as a function of $\mathrm{pH}$ between protein (emulsifier) and each polysaccharides used in the system, to determine the highest protein-polysaccharide electrostatic attraction. It should be noted that, the electrical properties of the first layer of multilayer emulsions are determined by the charge of the emulsifier, and they can thus be controlled by selecting different types of emulsifiers. Each of these emulsifiers have different electrical 
characteristics, which can influence the formation and properties of multilayer interfaces $\left[4^{* *}\right]$. There are three methods of preparation that have been developed to produce stable multilayer systems: (i) saturation method, (ii) centrifugation method, and (iii) filtration method [4**]. For all of the methods mentioned above the (i) saturation method is an easy and rapid way to prepare multilayer emulsions. This technique consists mainly in the determination of the concentration in which oil droplets of emulsion are completely surrounded by polyelectrolyte. According to Guzey and McClements [4**], the saturation concentration for a particular system has to be determined empirically (for example, using $\zeta$-potential measurements). For example, Burgos-Díaz and coworkers [15*] used the saturation method to prepare secondary emulsions using lupin protein isolate from AluProtCGNA and chitosan. Figure 3 shows that the $\zeta$-potential values of emulsions (at pH 5) increased significantly when the chitosan concentration was increased between 0.02 and 0.06 (wt \%), indicating that chitosan molecules were adsorbed onto lupin protein. Additionally, the net charge on the droplet changed from negative to positive as the chitosan concentration was increased until reaching a relatively constant value, which indicates that cationic chitosan molecules saturated the surfaces of the protein-stabilized droplets (saturation concentration). 


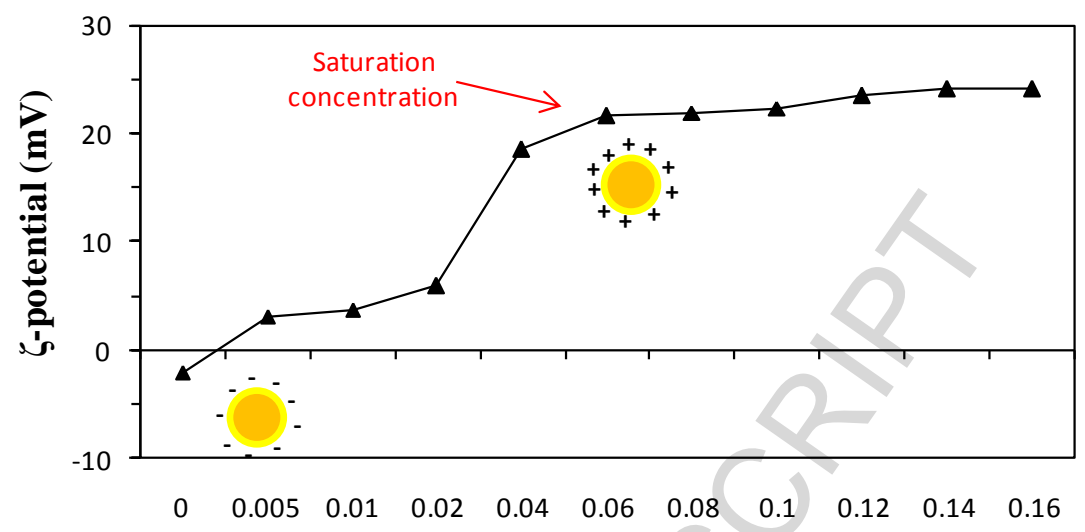

Chitosan (wt\%)

Figure 3: The particle electrical charge ( $\zeta$-potential) of biopolymer-coated lipid droplets in the secondary emulsions as a function of chitosan concentration at $\mathrm{pH}$. Figure adapted from Burgos-Díaz et al.[15*].

Benjamin and coworkers [17] also utilized saturation method for forming multilayer emulsions using $\beta$-lactoglubulin and pectin layers. The adsorption of pectin to $\beta$ lactoglubulin was indicated by the change in $\zeta$-potential from a positive potential $(\zeta=+64$ $\mathrm{mV}, \mathrm{pH} 3)$ to a negative potential $(\zeta=-11 \mathrm{mV}, \mathrm{pH} 3)$. Although the electrical potential on the droplets after pectin adsorption was relatively low $(\zeta=<20 \mathrm{mV})$, the emulsion remained stable due to the thicker interfacial layers which resulted in strong steric repulsions. Li and coworkers [18], used the saturation method to stabilize $\mathrm{O} / \mathrm{W}$ emulsions prepared with $\beta$ lactoglubulin, chitosan and pectin layers. The influence of chitosan concentration on the $\zeta$ potential was measured at different $\mathrm{pH}$ (4.5 and 5.5). At all $\mathrm{pH}$ values, the $\zeta$-potential became increasingly positive as the chitosan concentration was raised until a relatively constant value was attained, indicating that cationic chitosan molecules adsorbed to the surfaces of the protein-stabilized droplets until they became saturated with chitosan. 
It should be noted, that regardless of the method utilized for preparing emulsions, it is necessary to control the system composition and preparation conditions in order to form stable multilayer emulsions. Thus, it is very important to guarantee that: (1) there is sufficient polyelectrolyte present in the system to surround the whole surface of oil droplets; (2) there is not too much free polyelectrolyte in the aqueous phase to promote depletion flocculation of oil droplets; and (3) the polyelectrolyte adsorbs faster than the occurrence of the particle-particle collisions. Additionally, these processes depend on the size and concentration of the polyelectrolyte molecules and colloidal particles present, as well as on the solution conditions (e.g., $\mathrm{pH}$, ionic strength, dielectric constant, temperature, and stirring) [4**].

On the other hand, the (ii) centrifugation method requires the preparation of a solution with polyelectrolyte in excess to be added to the colloidal suspension. Centrifugation (one or more times) is employed to eliminate the non-adsorbed polyelectrolyte and, particles are collected and re-suspended in buffer. After, the next polyelectrolyte solution can be added. The possible particle aggregation, as consequence of centrifugation, can be the main problem of this method. During the (iii) filtration method, the colloidal suspension is mixed with polyelectrolyte solution in excess. Güzey and McClements [4**] mentioned that the utilization of the centrifugation method causes major problems of droplet aggregation. In these methods the non-absorbed polyelectrolyte molecules are eliminated by membrane filtration using filtration under pressure, which allows the polyelectrolyte molecules to pass through. If the volume is maintained with constant buffer solution addition, colloidal particles are non-forced to proximity and particle aggregation is not a problem. Accordingly, we believe that better results and fewer drawbacks are obtained when the 
Burgos-Díaz et al. /Current Opinion in Colloid \& Interface Science (2016)

saturation and filtration methods are employed for forming multilayer emulsions, both methods producing less droplet aggregation.

\section{Multilayer emulsion systems prepared using vegetable proteins and polysaccharides}

Vegetable proteins in alternation with polysaccharide can be effectively used for the design and formation of multilayer emulsions. For example, proteins constitute a special class of compounds, which are very suitable for $L b L$ technique. They are, on the one side, natural weak polyelectrolytes, but on the other side, several hydrophobic groups in their structure confer interfacial activity to these molecules. Thus, being applied as emulsifiers, the proteins simultaneously charge the adsorption interface, and enable the following steps of $L b L$ deposition either by other biopolyelectrolytes or by conventional polyelectrolytes [8].

In the last ten years, soy proteins have become the most used emulsifiers of plant origin (Table 1); most probably, due to the extensive production of this legume worldwide and the increased use of it in processed food. Although soy proteins have been found to be effective emulsifiers, the emulsions formed with these proteins alone are not very stable [19]. Therefore, complex coacervation with soy proteins and polysaccharides has been commonly used to improve stability [4**]. One of the most recurrent systems described in literature included the soy protein isolate (SPI), which is positively charged at $\mathrm{pH}$ values below its isoelectric point $(\mathrm{pI} \approx 4.6)[20,21]$, and several anionic polysaccharides such as alginate [5], pectin [22, 23], gum arabic [20, 24], octenyl succinate starch (OSA starch) [21, 25], flaxseed gum [26] and soy soluble polysaccharides (SSPS) [13, 27]. Additionally, since the SPI at $\mathrm{pH}$ values higher than its $\mathrm{pI}$ carried negative charge, it is also possible to produced emulsions where the droplets are coated with SPI and cationic polysaccharides such as gelatin [28] and chitosan [5]. Most of the recent studies are based on emulsions containing bilayer-coated droplets, but few also added a third layer, (i.e. SPI-OSA starch- 
chitosan) [25]. Multilayer-coated droplets, in general, are more stable than the bilayercoated ones $\left[4^{* *}, 25\right]$.

In all the cases, polysaccharides contributed to improving the long-term storage stability, broadening the $\mathrm{pH}$ range where the emulsion is stable, and giving resistance to environmental changes like ionic strength and temperature. It was also observed, in some of the studies, that the nature of the polysaccharide might determine the extent of the stabilization. Thus, when the effects of high methoxyl pectin and SSPS over the SPI stabilized emulsion were compared, it was observed that SSPS coating can enhance the stability of soy protein isolate emulsions at neutral $\mathrm{pH}$ and $\mathrm{pH}$ range of 3-4 [29]. These emulsions did not show creaming or flocculation after 15 days of storage, while pectin caused flocculation of the emulsion droplets via a depletion mechanism at neutral $\mathrm{pH}$, and extensive droplet aggregation at $\mathrm{pH}$ below 4.0 via bridge flocculation [29].

Other study analyzed the effect of different polysaccharides, gum arabic (GA), locust bean gum (LBG), xanthan gum (XG) and a mixture of XG-LBG, over the protein isolates of two species of bean (Phaseolus vulgaris and Phaseolus coccineous) [10]. The results indicated that the addition of XG or XG-LBG stabilizes emulsions better than $\mathrm{XBG}$, which in turn has a better effect than GA (for both concentrations studied $0.1 \% \mathrm{w} / \mathrm{v}$ and $0.25 \% \mathrm{w} / \mathrm{v}$ ). The stabilizing effect of $\mathrm{XG}$ is better than XG-LBG at higher polysaccharide concentrations. The stabilizing result is due to the creation of a network, protecting the oil droplets from coalescence.

The effect of cationic (chitosan) and anionic (alginate) polysaccharides were also compared, Zhang and coworkers reported that the droplets stabilized by alginate-SPI were stable to aggregation at $\mathrm{pH}$ values from 3 to 7 (without salt) and in salt concentration of 0 $500 \mathrm{mM} \mathrm{NaCl}(\mathrm{pH}$ 3). On the other hand, the droplets stabilized by chitosan-SPI exhibited 
Burgos-Díaz et al. /Current Opinion in Colloid \& Interface Science (2016)

good stability at pH 3-6 (without salt). However, droplets aggregation occurred in emulsions stabilized by chitosan-SPI at salt concentration $\geq 300 \mathrm{mM}(\mathrm{pH}$ 5). Other interesting difference was observed when the emulsions' digestibility was tested; chitosan coating was capable of reducing the lipids digestibility and bioaccessibility of carotenoids in emulsions, while alginate had no significant effect on the lipid digestion [5].

Differences were also observed in relation to the protein nature, for example, protein isolates from P.coccineous created emulsions with slightly larger oil droplets than $P$. vulgaris, irrespective of polysaccharide addition [10]. Other study compared the coacervates made up by the complexation of gum arabic (GA) and protein concentrates from flaxseed (FPC) and soy (SPC). The researchers observed that the addition of $2 \%$ GA to FPC- and SPC-stabilized emulsion would strengthen their stability by increasing the viscosity for FPC, and due to competitive adsorption for the SPC. However, the SPCstabilized emulsion in the presence of GA had better emulsion stability and better stability against environmental factors such as $\mathrm{NaCl}$ concentration and thermal treatments than FPC [19]. The results also indicate that the SPC-stabilized emulsions exhibit better stability when the SPC is partially denatured [19]. Along the same line, Ray and Rousseau's publication [30] showed that denatured soy whey proteins (dSWP) present better emulsifying activity than native SWP, and emulsion stability against coalescence of dilute O/W emulsions increases considerably by the addition of SSPS [30].

The majority of the studies using SPI presented here were aimed to the encapsulation of bioactive compounds such as casein hydrolysate [22], propolis [23], paprika oleoresin carotenoid [24], sweet orange oil [20] and vanillin [25]. All encapsulations were established using the $L b L$ polyelectrolyte deposition method and afterwards the emulsions were frozen or spray dried. The encapsulation proved to be a convenient way of achieving the different 
goals of each study, like attenuating the bitter taste of casein hydrolysate, controlling release of the compound in foods $[23,25]$ and protecting compounds from degradation [24] and flavor lost [20]. In addition, the incorporation of SPI may represent an improvement in the nutritional value of the preparations.

Other soy related compounds that are associated to complex coacervation and have been often used in emulsion stabilization are soy oil bodies. Oil bodies are lipid storage vesicles that are naturally found in many plant seeds. Oil bodies consist of a lipid core that is coated by a layer of oleosin proteins and phospholipids [31]. The main application for these organelles is emulsion stabilization and, since they occur naturally and do not require additional emulsifiers or homogenization procedures, can lead to more sustainable and environmentally friendly processing operations of food and cosmetics [31, 32]. However, it is known that oil bodies present poor physical stability to particle aggregation, thus complex coacervation with an additional polysaccharide layer was a successful approach to improve stability. The polysaccharides used in these studies were negatively charged and include pectin and $\kappa, 1, \lambda$-carrageenan $[31,32,33,34]$. One group also reported that enzymatic cross-linking of the polysaccharide can further stabilize the system in an extended $\mathrm{pH}$ range [32].

The system observed for maize germ oil bodies [35] is similar to the latter. Oil bodies in the form of a natural oil-in-water emulsion cream can be obtained at a relatively high yield from maize germ. The very high stability of oil bodies in the recovered cream was attributed to the presence of an adsorbed mixed surface membrane around the triglyceride core, which was believed to be made up of a primary mixed layer of phospholipids and oleosins, and a secondary layer of extraneous germ proteins. However, oil bodies extracted from maize germ tend to aggregate strongly, thus the natural emulsions, prepared by 
diluting the oil body cream into relatively low oil content, exhibit very low storage stability against creaming. Again here, the authors found that the addition of the xanthan gum polysaccharide enhances the stability of the emulsions and the result was more distinct when the oil bodies carried at their surface showed appreciable amounts of extraneous maize germ proteins [35].

The second protein isolate most encountered in literature is the one obtained from pea. Several authors described multilayer emulsions made from pea protein isolate (PPI) and polysaccharides such as gum arabic [36] and pectin [1, 7, 37]. Most of the emulsions were prepared using the $L b L$ method $[1,7,37]$, but one study compared two different methods the $L b L$ deposition (called two-steps method) and the pre-mixed of the PPI with the GA before the oil was added (namely one-step method) [36]. They found that the two-steps method improves the biopolymer stability at the interface in front of PPI alone, but less than in the one-step method. In general, the addition of a polysaccharide to the primary emulsion with the protein isolate improves the stability of the emulsions to ageing, $\mathrm{pH}$ changes, and can also increase the resistance to depletion flocculation caused by maltodextrin addition $[1,36]$. The PPI-pectin coacervate was successfully used for the microencapsulation of food flavors [7] and polyunsaturated fatty acids (PUFA)-rich oil [37] aiming, in both cases, for the protection against degradation and oxidation of the bioactive compounds.

Other alternative plant protein isolates have been recently used. One of them was the potato protein isolate. Oil in water emulsions containing this protein isolate exhibited poor stability (no longer than 1 day after preparation); therefore, the addition of chitosan was tested resulting in improved stability as observed in the previously mentioned coacervates [38]. 
Burgos-Díaz et al. /Current Opinion in Colloid \& Interface Science (2016)

A very promising alternative is the protein isolate obtained from a novel lupin variety, AluProt-CGNA. This new lupin variety was developed offering a higher protein concentration (60\% in dehulled seeds) than soy with an amino acid profile which rivals the current star of plant proteins [39]. It has been shown that lupin protein isolate (LPI) has good emulsifying properties and, as the soy counterpart, the LPI-coated emulsions can be further stabilized by the addition of polysaccharide $\left[15^{*}\right]$. The authors investigated the effects of the usage of a secondary layer of chitosan and a third of xanthan gum. The primary emulsions (AluProt-CGNA protein-coated oil droplets) were highly unstable to aggregation at $\mathrm{pH}$ values around their isolectric point $(\mathrm{pH} \approx 4.6)$ and temperature $\left(30-90^{\circ} \mathrm{C}\right)$. The emulsions stabilized with protein isolate from AluProt-CGNA, chitosan, and xanthan gum membranes, however, presented greater physical stability than the emulsion stabilized only with lupin protein. The droplets in the secondary and tertiary emulsions had good stability to aggregation over a wide range of $\mathrm{pH}$ values, temperature, and salt concentrations. The tertiary emulsions seem to offer higher stability than the ones with two layers.

Individual plant proteins have also been used to form multilayer emulsions with polysaccharides. In these cases, the main goal was the characterization of the system and determination of the prerequisite protein features, which are involved in the coacervate interfacial properties. Although the use of individual proteins creates a good and controlled interaction system, the up-scale of the process becomes costlier than protein isolates. Ducel and coworkers investigated the emulsifying properties of four plant proteins comparing the surface activity of wheat alpha gliadin, pea globulin, soy globulin, and pea albumin [40]. They found out that alpha gliadin and pea globulin have the highest surface active properties at the oil-water interface, but both work through different mechanisms. Alpha 
gliadin interfacial behavior is characterized by a pronounced viscoelasticity, whereas pea protein interfacial behavior became elastic after a long initial adsorption period. After these two proteins were tested in complex coacervation with gum arabic, the authors could observe that complex coacervates formed under appropriate conditions tend to decrease the interfacial tension more strongly than the plant protein solution alone [41]. They also showed that pea globulin adsorption is mainly influenced by concentration, and in the case of alpha-gliadin the interfacial tension was found to be dependent on the $\mathrm{pH}$ of the solution. Another group tested the effect of two different polysaccharides, xanthan gum and pectin, on the fish oil-in-water gliadin-stabilized emulsions [3]. They found out that the addition of xanthan gum increased the oxidative stability, and on the other hand, the addition of pectin actually decreased their oxidative stability. Nevertheless, in both cases the addition of anionic polysaccharides led to faster rate of lipid digestion in the gliadin-stabilized emulsions.

Other aspect that was considered was the mechanism of emulsion formation employing homogenization as the second step to produce stable $L b L$ emulsion [42]. To this aim they used fish oil in water emulsions, encapsulated with a positively charged inner soy $\beta$ conglycinin (7S) layer by high shear mixing, followed by deposition of a negatively charged outer high methoxyl pectin (HMP) layer using homogenization (500 or 3000 psi). The results of this investigation showed that the one layer emulsion produced by high shear mixing was less stable, because the droplets size was too large, which led to creaming and subsequent coalescence and aggregation. Stable emulsions were produced by high shear mixing followed by homogenization after polysaccharide deposition, with significantly lower droplet size. The best pressure for homogenization was 500 psi rendering a uniform two layer shell thickness [42]. 
Burgos-Díaz et al. /Current Opinion in Colloid \& Interface Science (2016)

Table 1: Main published experimental studies on vegetable protein-polysaccharide electrostatic combination to stabilize emulsions

\begin{tabular}{|c|c|c|}
\hline Protein & Polysaccharide & Reference \\
\hline Soy oleosins & Pectin & {$[31]$} \\
\hline Soy oleosins & Beet pectin & [32] \\
\hline Soy oleosins & $\kappa, 1, \lambda$-carrageenan & [33] \\
\hline Soy oleosins & 1-carrageenan & [34] \\
\hline Soybean protein isolate & $\begin{array}{c}\text { High methoxyl pectin and soy } \\
\text { polysaccharides }\end{array}$ & [29] \\
\hline Soybean protein isolate & Pectin & [22] \\
\hline Soybean protein isolate & Pectin & [23] \\
\hline Soybean protein isolate & Gelatin & [28] \\
\hline Soybean protein isolate & Flaxseed gum & [26] \\
\hline Soybean protein isolate & Gum arabic & [24] \\
\hline Soybean protein isolate & Gum arabic & [20] \\
\hline Soybean protein isolate & polysaccharides & [13] \\
\hline Soybean protein isolate & Soy polysaccharides & [27] \\
\hline Soybean protein isolate & succinate starch/chitosan & [21] \\
\hline Soybean protein isolate & Octenyl succinate starch/chitosan & [25] \\
\hline Soybean protein isolate & Alginate and chitosan & [5] \\
\hline Soy whey protein (denaturated) & Soy polysaccharides & [30] \\
\hline $\begin{array}{c}\text { Flaxeed protein and soybean } \\
\text { protein concentrate }\end{array}$ & Gum arabic & [19] \\
\hline Soy $\beta$-conglycinin & Methoxyl pectin & [42] \\
\hline Pea protein isolate & Gumarabic & [36] \\
\hline Pea protein isolate & Pectin & [1] \\
\hline Pea protein isolate & Pectin & [7] \\
\hline Pea protein isolate & Pectin & [37] \\
\hline$\alpha$-gliadin (wheat)-Pea globulin & Gum arabic & [41] \\
\hline Wheat protein & Xanthan gum and pectin & [3] \\
\hline Maize germ & Xanthan gum & [35] \\
\hline $\begin{array}{c}\text { Bean and scarlet runner bean } \\
\text { protein isolates }\end{array}$ & $\begin{array}{c}\text { Gum Arabic, gum locust bean and } \\
\text { xanthan gum }\end{array}$ & [10] \\
\hline Potato protein isolate & Chitosan & [38] \\
\hline Lupin protein isolate & Chitosan and xanthan gum & {$\left[15^{*}\right]$} \\
\hline
\end{tabular}


Burgos-Díaz et al. /Current Opinion in Colloid \& Interface Science (2016)

\section{Potentials applications of multilayer emulsion}

Finally, it is very important to mention that multilayer emulsions may have a number of potential applications in food industry and in the medical and cosmetic fields. Their composition and properties can help stabilize oil droplets against aggregation caused by exposure to environmental stresses, such as $\mathrm{pH}$, salt, thermal processing, chilling, freezing, dehydration, and mechanical agitation [43*]. On the other hand, bioactive lipophilic compounds can also be protected from chemical degradation by the incorporation of a multilayer emulsion in their inner oil phase, thereby isolating them from other watersoluble ingredients and giving them protection against environmental stresses. The incorporation of bioactive compounds to food (nutraceuticals) may have physiological benefits and reduce de risk of diseases. However, its incorporation represents a big challenge. Multilayer emulsions may confer protective mechanisms required in food formulation to these molecules. Polyunsaturated fatty acids-rich oils are actually considered a functional food, but their low solubility, high oxidation risk and undesirable flavor hinder its use. Emulsification with pea protein isolate and pectin, and posterior microencapsulation by spray drying, may be a way to allow its use and commercialization [37]. Additionally, multilayer particles prolong compound residence time in the gastrointestinal tract and increase the surface available to interact with tissues [44]. The properties of the multilayers can often be made to change in response to specific environmental triggers, such as dilution, $\mathrm{pH}$, ionic strength, temperature, or enzyme activity. For instance, the chemical stability of emulsified lipids can be improved by minimizing interactions between the encapsulated lipids and chemically reactive aqueous phase substances by controlling the interfacial charge and thickness, e.g. the oxidative stability of $\omega-3$ fatty acids can be improved by preventing transition metals from coming into close contact with them [45]. 
Burgos-Díaz et al. /Current Opinion in Colloid \& Interface Science (2016)

The same approach can be used to increase the chemical stability of other labile lipophilic components, e.g., carotenoids, vitamins, flavors [7, 24].

Low solubility in water tends to be an intrinsic property of many promising drug candidates and in consequence they have failed to enter clinical development processes because of solubility problems. The incorporation of lipophilic drugs in $\mathrm{O} / \mathrm{W}$ emulsions is a mode to overcome solubility problems. The release rate of encapsulated components from oil droplets can be controlled by designing the thickness and permeability of multilayer coatings surrounding them $\left[43^{*}\right]$. The formation of $L b L$ polyelectrolyte capsules was applied to prepare sustainable release formulations of some water soluble drugs as ibuprofen, furosemide and insulin, which under normal circumstances completely dissolve in few minutes or nanocolloids of insoluble drugs (tamoxifen and paclitaxel) with very high drug content (up to 90\%) preventing aggregation and controlling drug release [46]. Results obtained indicated a clear interest of multilayer emulsions to increase and control drug delivery. Obviously, the most convenient route for the systemic delivery of pharmaceuticals is oral, but, bioavailability via this route is poor, as a consequence of susceptibility to acid, enzymatic hydrolysis or bacterial fermentation. The fact that oral delivery formats are more appealing in terms of convenience and patient preference will continue to drive further research and development of multilayer emulsions [47].

With the improvement of people living standard the demand for sophisticated and costly personal care products has increased, which induces the cosmetic scientists to develop high technological formulations with high quality and presentation [48, 49]. The most common used bases for skincare products are emulsions. Certain penetration of the active compound into the skin is required to obtain a cosmetic effect after topical application, making boundary between topical pharmaceutics very narrow [49]. At present, conventional 
emulsions are the most widely used system in the industry [43*]. However, structured emulsions such as multilayer emulsions, stabilized by vegetable proteins and polysaccharide may be advantageous for certain applications. Its study and development can benefit us in aspects of daily life.

Despite their potentials applications and advantages, it is important to mention that there are certain disadvantages that may limit the widespread commercialization of multilayer emulsions [43*]. With this type of system it is necessary to have a precise control over system composition and preparation procedures in order to avoid droplet aggregation through bridging, depletion and other effects [4**, 50]. The high susceptibility for droplet aggregation occuring during preparation puts a limit on the maximum amount of oil that can be coated using this approach. Additional ingredients (e.g., biopolymers for coatings) and processing steps (e.g., mixing) are required to prepare multilayer emulsions compared to conventional emulsions. Consequently, this type of system is more expensive and difficult to prepare, but the potential benefits (improved functionality or extended shelf life) can outweigh the costs for certain applications.

\section{Conclusions}

In this review, it has been summarized the usage of plant proteins on the formation and utilization of multilayer emulsions. Emulsions are applied in different fields of human activity, such as food industry, pharmaceuticals, cosmetics and medicine; and therefore, its stability and protection against colloidal degradation or environmental stress is a challenge for producers. O/W emulsions are widely used for encapsulating bioactive compounds, increasing their solubility and stability. The increasing consumer concern over safety of animal products points to the advantage of using vegetable proteins as emulsifiers. The use 
of vegetable proteins as emulsifiers reflects the actual "green" tendency in food, pharmaceutical and cosmetics industries.

To overcome emulsion instability, a barrier can be created at the $\mathrm{O} / \mathrm{W}$ interface, hence protecting the emulsion from breakage. Additional coating layers of a suitable combination of biopolymers (polyelectrolyte) as stabilizers can be deposited using the $L b L$ electrostatic technique. $L b L$ deposition at oil droplet surfaces in $\mathrm{O} / \mathrm{W}$ emulsions systems seems to be easily adapted to production conditions in industrial formulations because of its simplicity and relative low cost. The plant origin emulsifiers mostly used are proteins of soy, pea, and potato; however, new alternatives have been developed such as the promising novel lupin variety AluProt-CGNA. Some polysaccharides as alginate, pectin, gum arabic, flaxseed gum, chitosan can be cited as stabilizers. The following advantages of $L b L$ approach for forming multilayer emulsions can be summarized so far: (i) easy manufacturing, (ii) decreased lipid oxidation, (iii) good delivery system for high lipophilic active principle, (iv) biocompatibility and biodegradability, (v) degradable by digestible enzymes, and (vi) abundant renewable sources.

Vegetable proteins can be used to prepare matrices, which can be tailored for a specific application in innovative products. The high versatility of this method allows it to create advanced systems in materials release. However, more studies are required to ensure the ideal design. Vegetable proteins are an interesting promise for creating novel systems with the potential to encapsulate bioactive compounds with controlled release via oral route. Multilayer emulsions have better stability to environmental stresses than conventional emulsions under certain conditions. Nevertheless, more research is still needed to establish, at a fundamental level, the factors that influence the preparation of stable multilayered emulsions with specific functional attributes, including emulsifier characteristics. 


\section{Acknowledgements}

This review was supported by CONICYT through FONDECYT project $\mathrm{N}^{\circ} 3140001$.

\section{References}

[1] Gharsallaoui A, Saurel R, Chambin O, Cases E, Voilley A, Cayot P. Utilisation of Pectin Coating to Enhance Spray-Dry Stability of Pea Protein-Stabilised Oil-in-Water Emulsions. Food Chem 2010; 122:447-54.

[2] *Nesterenko A, Alric I, Oise Silvestre F, Durrieu V. Vegetable Proteins in Microencapsulation: A Review of Recent Interventions and Their Effectiveness. Industrial Crop Prod 2013; 42: 469-79.

[3] Qiu C, Zhao M, McClements D.J. Improving the Stability of Wheat Protein-Stabilized Emulsions: Effect of Pectin and Xanthan Gum Addition. Food Hydrocolloids 2015; 43:377-87.

[4] **Güzey D, Mcclements D.J. Formation, stability and properties of multilayer emulsions for application in the food industry. Adv Colloid Interface 2006; 128-130:227-48.

[5] Zhang C, Xu W, Jin W, Shah B, Li Y, Li B. Influence of Anionic Alginate and Cationic Chitosan on Physicochemical Stability and Carotenoids Bioaccessibility of Soy Protein Isolate-Stabilized Emulsions. Food Res Inter 2015; 77:419-25.

[6] Ogawa S, Decker EA, McClements DJ. Influence of environmental conditions on the stability of oil in water emulsions containing droplets stabilized by lecithin-chitosan membranes. J Agric Food Chem 2003; 51(18):5522-7.

[7] Gharsallaoui A., Roudaut G., Beney L., Chambin O., Voilley A., Saurel R. Properties of Spray-Dried Food Flavours Microencapsulated with Two-Layered Membranes: Roles of Interfacial Interactions and Water. Food Chem 2012; 132:1713-20.

[8] Grigoriev D.O, Miller R. Mono- and multilayer covered drops as carriers. Curr Opin Colloid Interface Sci 2009; 14:48-59.

[9] Burgos-Díaz, C., Rubilar, M., Morales, E., Medina, C., Acevedo, F., Marqués, A.M., Shene, C. (2015). Naturally occurring protein-polysaccharide complexes from linseed (Linum usitatissimum) as bioemulsifiers. Eur J Lipid Sci Technol 118; 165-174.

[10] Makri E.A, Doxastakis G.I, Study of emulsions stabilized with Phaseolus vulgaris or Phaseolus coccineus with the addition of Arabic gum, locust bean gum and xanthan gum. Food Hydrocolloids 2006; 20:1141-1152. 
[11] Hu M, McClements DJ, Decker E.A. Lipid Oxidation in Corn Oil-in-Water Emulsions Stabilized by Casein, Whey Protein Isolate, and Soy Protein Isolate. J Agric Food Chem 2003; 51(6):1696-1700.

[12] *McClements D.J. Protein-Stabilized Emulsions. Curr Opin Colloid Interface Sci 2004; 9: $305-13$.

[13] Yin B, Deng W, Xu K, Huang L, Yao P. Stable Nano-Sized Emulsions Produced from Soy Protein and Soy Polysaccharide Complexes. J Colloid Interface Sci 2012; 380: 51-59.

[14] Diftis N, Kiosseoglou V. Stability against Heat-Induced Aggregation of Emulsions Prepared with a Dry-Heated Soy Protein Isolate-dextran Mixture. Food Hydrocolloids 2006; 20(6):787-92.

[15] *Burgos-Díaz C, Gallardo M, Morales E, Piornos J, Marqués A.M, Rubilar M. Utilization of proteins from AluProt-CGNA (a novel protein-rich lupin variety) in the development of oil-in-water (O/W) multilayer emulsion systems. Eur J Lipid Sci Technol 2016; 117:. doi: 10.1002/ej1t.201500260.

[16] Perrechil F.A., Cunha R.L. Stabilization of Multilayered Emulsions by Sodium Caseinate and $\kappa$-Carrageenan. Food Hydrocolloids 2013; 30(2):606-13.

[17] Benjamin O, Silcock P, Leus M, Everett D.W. Multilayer Emulsions as Delivery Systems for Controlled Release of Volatile Compounds Using $\mathrm{pH}$ and Salt Triggers. Food Hydrocolloids 2012; 27:109-18.

[18] Li Y, Hu M, Xiao H, Du Y, Decker E.A, McClements D.J. Controlling the functional performance of emulsion-based delivery systems using multi-component biopolymer coatings. Eur J Pharm Biopharm 2010; 76 (1):38-47.

[19] Wang B, Wang L-J, Li D, Adhikari B, Shid J. Effect of Gum Arabic on Stability of Oil-inWater Emulsion Stabilized by Flaxseed and Soybean Protein. Carbohyd Polym 2011; 86:34351.

[20] Jun-xia X, Hai-yan Y, Jian Y. Microencapsulation of Sweet Orange Oil by Complex Coacervation with Soybean Protein Isolate/gum Arabic. Food Chem 2011; 125(4):1267-72.

[21] Noshad M, Mohebbi M, Shahidi F, Koocheki A. Freezeethaw Stability of Emulsions with Soy Protein Isolate through Interfacial Engineering. Int J Refrigeration 2015; 58:253-60.

[22] Mendanha D.V, Molina Ortiz S.E, Favaro-Trindade C.S, Mauri A, Monterrey-Quintero E.S, Thomazini M. Microencapsulation of Casein Hydrolysate by Complex Coacervation with SPI/pectin. Food Res Int 2009; 42:1099-1104. 
[23] Nori M.P, Favaro-Trindade C.S, de Alencar S.M, Thomazini M, de Camargo Balieiro J.C, Contreras Castillo C.J. Microencapsulation of Propolis Extract by Complex Coacervation. LWT - Food Sci Technol 2011; 44:429-35.

[24] Rascón M.P, Beristain C.I, García H.S, Salgado M.A, Carotenoid retention and storage stability of spray-dried encapsulated paprika oleoresin using gum Arabic and Soy protein isolate as wall materials. LWT - Food Sci Technol 2011; 44:549-557.

[25] Noshad M, Mohebbi M, Shahidi F, Koocheki A. Effect of layer-by-layer polyelectrolyte method on encapsulation of vanillin. Int J Biol Macromol 2015; 81:803-808.

[26] Wang Y, Li D, Wang L-J. Adhikari B. The effect of addition of flaxseed gum on the emulsion properties of soybean protein isolate (SPI). J Food Eng 2011; 104:56-62.

[27] Tran T, Rousseau D. Stabilization of Acidic Soy Protein-Based Dispersions and Emulsions by Soy Soluble Polysaccharides. Food Hydrocolloids 2013; 30(1):382-92.

[28] Favaro-Trindade A.S, Santana A.S, Monterrey-Quintero E.S, Trindade M.A, Netto F.M. The Use of Spray Drying Technology to Reduce Bitter Taste of Casein Hydrolysate. Food Hydrocolloids 2010; 24:336-40.

[29] Roudsari M, Nakamura A, Smith A, Corredig M. Stabilizing Behavior of Soy Soluble Polysaccharide or High Methoxyl Pectin in Soy Protein Isolate Emulsions at Low pH. J Agr Food Chem 2006; 54:1434-1441.

[30] Ray M, Rousseau D. Stabilization of Oil-in-Water Emulsions Using Mixtures of Denatured Soy Whey Proteins and Soluble Soybean Polysaccharides. Food Res Int 2013; 52:298-307.

[31] Iwanaga D, Gray D, Decker E.A, Weiss J, McClements D.J. Stabilization of Soybean Oil Bodies Using Protective Pectin Coatings Formed by Electrostatic Deposition. J Agr Food Chem 2008; 56:2240-2245.

[32] Chen B, McClements D.J, Gray D.A, Decker E.A. Stabilization of Soybean Oil Bodies by Enzyme (Laccase) Cross-Linking of Adsorbed Beet Pectin Coatings. J Agric Food Chem 2010; 58:9259-65.

[33] Wu N-N, Yang X-Q, Teng Z, Yin S-W, Zhu J-H, Qi J-R. Stabilization of soybean oil body emulsions using $\kappa, 1, \lambda$-Carrageenan at different pH values. Food Res Int 2011; 44(4): 105968.

[34] Wu N-N, Huang X, Yang X-Q, Guo J, Yin S-W, He X-T, Wang L-J, Zhu J-H, Qi J-R, Zheng E-L. In Vitro Assessment of the Bioaccessibility of Fatty Acids and Tocopherol from Soybean Oil Body Emulsions Stabilized with I-Carrageenan. J Agr Food Chem 2012; 60: $1567-75$. 
[35] Nikiforidis C.V, Kiosseoglou V. Physicochemical Stability of Maize Germ Oil Body Emulsions as Influenced by Oil Body Surface-Xanthan Gum Interactions. J Agr Food Chem 2010; 58:527-32.

[36] Liu, S, Elmer C, Low N.H, Nickerson M.T. Effect of pH on the Functional Behaviour of Pea Protein Isolate-gum Arabic Complexes. Food Res Int 2010; 43(2):489-95.

[37] Aberkane L, Gaëlle R, Rémi S. Encapsulation and Oxidative Stability of PUFA-Rich Oil Microencapsulated by Spray Drying Using Pea Protein and Pectin. Food Bioprocess Technol $2014 ; 4: 1505-17$.

[38] Calero N, Muñoz J, Cox P.W, Heuer, A, Guerrero, A. Influence of chitosan concentration on the stability, microstructure and rheological properties of $\mathrm{O} / \mathrm{W}$ emulsions formulated with high-oleic sunflower oil and potato protein. Food Hydrocolloids 2013; 30:152-162.

[39] Piornos J.A, Burgos-Díaz C, Ogura T, Morales E., Rubilar M, Maureira-Butler I, SalvoGarrido H. Functional and physicochemical properties of a protein isolate from AluProtCGNA: A novel protein-rich lupin variety (Lupinus luteus). Food Res Int 2015; 76(3):716724.

[40] Ducel V, Richard J, Popineau Y, Boury F. Adsorption Kinetics and Rheological Interfacial Properties of Plant Proteins at the Oil-Water Interface. Biomacromolecules 2004; 5:20882093.

[41] Ducel V, Richard J, Popineau Y, Boury F. Rheological Interfacial Properties of Plant Protein-Arabic Gum Coacervates at the Oil-Water Interface. Biomacromolecules 2005; 6: 790-796.

[42] Xiang N, Lyu Y, Narsimhan G. Characterization of fish oil in water emulsion produced by layer by layer deposition of soy \& beta;-Conglycinin and high methoxyl pectin. Food Hydrocolloids 2016; 52: 678-89.

[43] *McClements D. Advances in Fabrication of Emulsions with Enhanced Functionality Using Structural Design Principles. Curr Opin Colloid Interface Sci 2012; 17: 235-45.

[44] Chen L, Remondetto G.E, Subirade M. Food protein-based materials as nutraceutical delivery systems. Trends Food Sci Tech 2006; 17:272-283.

[45] Gudipati V, Sandra S, McClements D.J, Decker E.A. Oxidative Stability and in Vitro Digestibility of Fish Oil-in-Water Emulsions Containing Multilayered Membranes. J Agr Food Chem 2010; 58(13):8093-99. 
[46] Agarwal A, Lvov Y, Sawant R, Torchilin V. Stable nanocolloids of poorly soluble drugs with high drug contant prepared using the combination of sonication and layer-by-layer technology. J Control Release 2008; 128:255-260.

[47] Truong-Le V., Lovalenti P.M., Abdul-Fattah A.M. Stabilization challenges and formulation strategies associated with oral biological drug delivery systems. Adv Drug Deliver Rev 2015; 93:95-108.

[48] Nazir H, Zhang W, Liu Y, Chen X, Wang L, Nasser M.M, Ma G. Silicone oil emulsions: strategies to improve their stability and applications in hair care products. Int Journal Cosmetic Sci 2014; 36:124-133.

[49] Yukuyama M.N., Ghisleni D.D.M., Pinto T.J.A., Bou-Chacra N.A. Nanoemulsion: process selection and application in cosmetics- a review. Int J Cosmetic Sci 2016; 38:13-24.

[50] Cho Y-H, McClements D.J. Theoretical Stability Maps for Guiding Preparation of Emulsions Stabilized by Protein-Polysaccharide Interfacial Complexes. Langmuir 2009; 25(12):6649-57. 
Burgos-Díaz et al. /Current Opinion in Colloid \& Interface Science (2016)

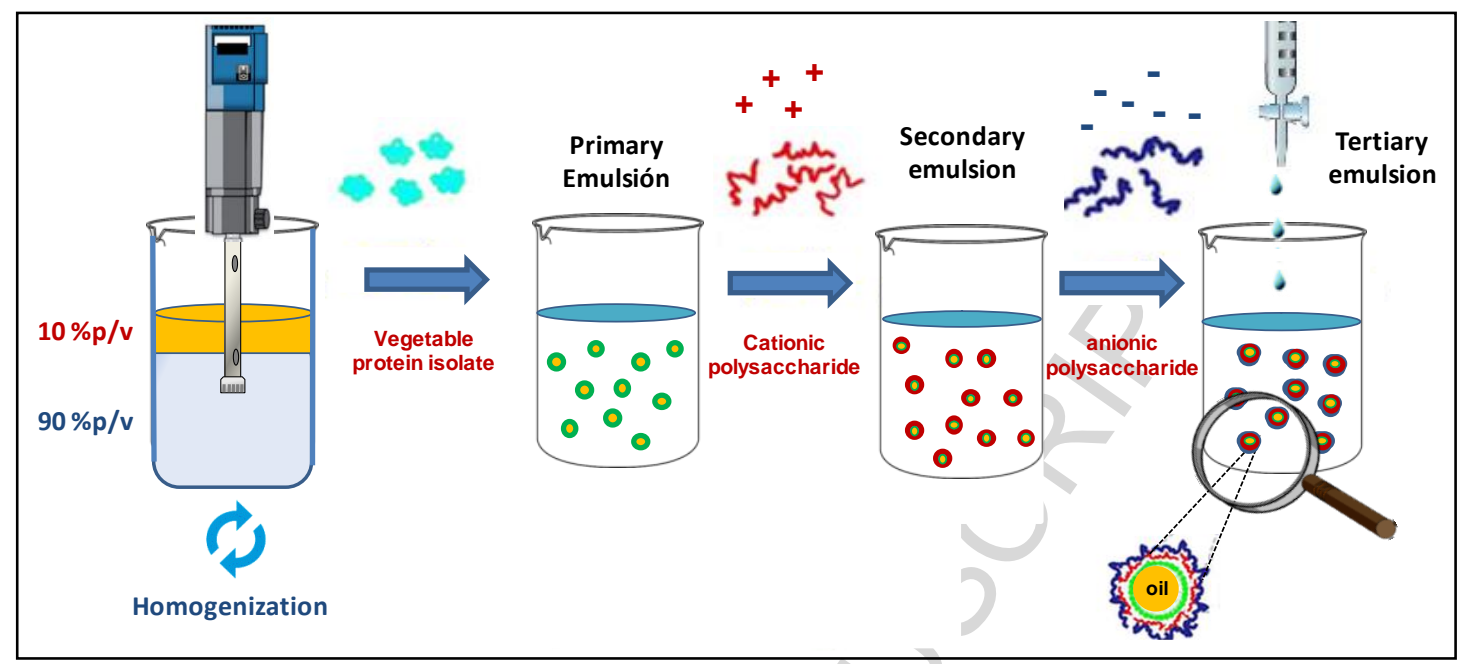

Graphical abstract 


\section{$\underline{\text { Highlights }}$}

- Multilayer emulsions have better stability to droplet flocculation and aggregation

- Multilayer emulsions can be prepared by using vegetable proteins

- Vegetable proteins are biopolymers with interesting functional properties

- Multilayer emulsions have potential applications in different industrial fields 